\title{
FAULT DETECTION OF A GAS TURBINE FUEL ACTUATOR BASED ON QUALITATIVE CAUSAL MODELS
}

\author{
T. Escobet*, L. Travé-Massuyès ${ }^{\dagger}$, S. Tornil*, J. Quevedo* \\ * UPC and LEA-SICA. \\ Edifici Vapor Sala, TR11. Rambla Sant Nebridi, 10 \\ 08222 Terrassa, Spain \\ Fax: +34937398628 \\ e-mail: \{teresa; stornil;joseba\}@esaii.upc.es \\ ${ }^{\dagger}$ LAAS-CNRS and LEA-SICA \\ 7 Avenue du Colonel-Roche \\ 31077 Toulouse, France \\ e-mail: louise@laas.fr
}

Keywords: Quantitative and Qualitative Methods of Fault Diagnosis. Fault and Uncertainty Modelling in Dynamical Systems.

\begin{abstract}
This paper presents an application of the Ca En model-based diagnosis software to a GE Frame 6 gas turbine. The paper focuses on the fault detection task and presents a mixed strategy, which combines an observer strategy with a simulation strategy, to achieve a good robustness/sensitivity trade-off. The presented application results have been obtained by running Ca En in real-time on the GE Frame 6 turbine owned by National Power at Aylesford (UK).
\end{abstract}

\section{Introduction}

Gas turbines are one of the prime movers for industrial plants and power generation. They are very complicate devices, particularly the larger ones generating over 200 Mega-Watts of electricity. Gas turbines are very critical pieces of the plants, for that they must be carefully monitored to detect problems at early stage, to be able to understand the state of the turbine, to know when maintenance should be performed, and to be able to rapidly diagnose the cause of a trip so that power can be restored as quickly as possible.

The TIGER product which resulted from the Esprit III Project 6862, named TIGER [5] is a monitoring system for gas turbines. It is connected directly to the gas turbine controller, and receives data at once per second, assessing the turbine conditions. This provides continuous condition assessment to support condition based maintenance, and performs fast diagnosis of abnormal situations. The Ca-En causal qualitative model based diagnosis module, was recently included in TIGER within the framework of the Trial Applications Esprit project 27548, named TIGER Sheba. Ca En expands the fault detection mechanism with the cross relationships between variables used in a mechanism for generating adaptive thresholds. The fault detection strategy of Ca En is twofold, switching from a closed-loop mode to an open-loop mode.

During the project, Ca En was demonstrated to work successfully for several gas turbine subsystems. At the end of this project, the Ca En tool was fully integrated with the Tiger system and performed continuous qualitative model based prediction and fault detection on a once per second basis. The TIGER Sheba project hence demonstrated on a real world, commercial scale problem, that the model based techniques represented by the $\mathrm{Ca} \sim \mathrm{En}$ tool work for practical user requirements.

The paper is organised along the following structure. Section 2 presents the gas turbine domain and the gas fuel system case study. Section 3 presents de Ca En strategy for fault detection whereas section 4 develops the Ca En model for the case study. Finally, section 5 and 6 include the results and conclusions.

\section{Gas turbine fuel actuator}

\subsection{Simplified diagram of the gas turbine}

The gas turbine simplified diagram, including the main components and variables, is given by Figure 1 below.

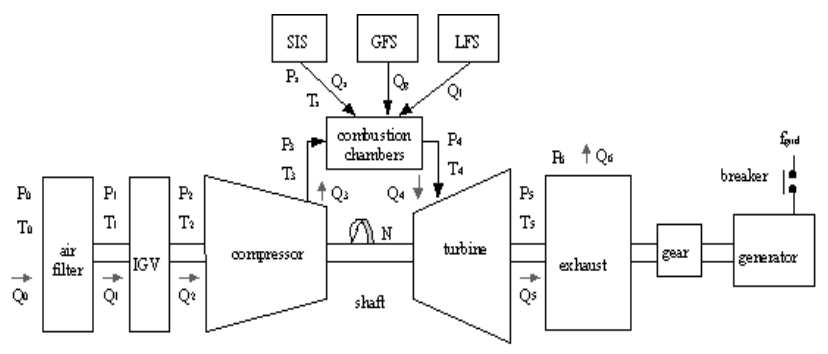

Figure 1. Gas turbine components

The gas turbine control system controls the shaft speed, modifying the gas fuel flow reference. For this reason, one of the critical parts of the turbine is the Gas Fuel System supply (GFS). Indeed, the performance and efficiency of the turbine 
highly depends on an accurate control of the fuel input. This is just the task of the GFS.

\subsection{Gas Fuel Supply}

The main components of the GFS are two actuators: the Stop Ratio Valve (SRV) and the Gas Control Valve (GCV). These valves are series connected and control the flow of gas fuel that enters in the combustion chambers. The first of these valves, the SRV, is controlled by a feedback loop that maintains constant the gas pressure at its output (pressure between the two valves) fpg2. This pressure being constant, the gas fuel flow is just determined by the position of the GCV. Hence, the GCV is a controlled valve position.

The SRV and GCV valves, and their associated feedback loops are shown in the Figure 2.

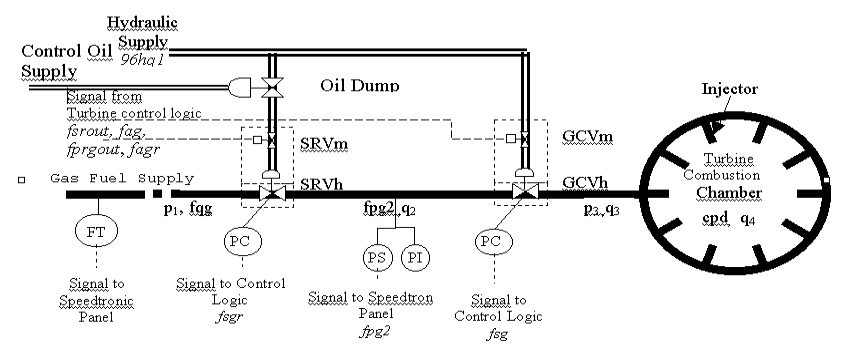

Figure 2. Gas fuel supply schema

The GFS system is monitored by means of three sensors that measure:

- the two valve positions $f_{s} g r$ and $f_{s} g$;

- $\quad$ the pressure between the two valves fpg2.

The two valve set-points correspond to the signals fprgout and fsrout which are provided by the controller.

\subsection{GFS fault detection requirements}

The turbine and its sub-systems are directly linked to a control system, which continuously compensates for faults and disturbances. As a result of this compensation, faults generally manifest during transient periods and for a short amount of time. The fault detection sensitivity is hence critical. However, as usual, a compromise must be found in order to be robust enough with respect to the noisy environment.

In particular, one of the main concerns of the users is the detection of sudden changes in the fuel valve position (spikes) which manifest during one sample only (cf. section 5), being one second the data acquisition period. This requires sufficiently precise detection models, which carefully track the system behaviour and base the fault detection mechanism on adaptive thresholds (cf. section 3).

From the discussion with the users and turbine designers, it appeared that some abnormal situations might as well be caused by external factors (disturbances). In this work, our focus is not put on such faults but rather on physical component breakdowns.

\section{A semi-closed-loop strategy for fault detection based on qualitative causal models}

Ca En [9] is a model-based supervision system devoted to complex dynamic systems. Ca En's representation formalism allows one to combine empirical causal knowledge and first principles of the domain. Ca En has two processing modules (cf. [10] for more details about the algorithms):

- A fault detection module based on a causal interval prediction mechanism;

- A fault isolation module based on an abstraction of the models in terms of a temporal causal model.

This paper restricts its scope to the fault detection module. It shows that the Ca En fault detection strategy can be viewed as a mixed strategy, combining an observer type strategy with an open-loop simulation strategy to determine the residuals and assess variable misbehaving. We call this strategy a semiclosed loop strategy (SCL).

\subsection{The Ca $\sim$ En diagnosis system knowledge representation formalism}

The Ca En formalism is based on a two-level representation scheme for the description of a physical system:

- a causal model in which the links represent the causal influences existing among the variables, referred as the local level;

- an analytical equation level which allows one to represent algebra-differential equations, referred as the global level.

Both levels can manage imprecise knowledge. A Ca En program represents a formal model of the physical system built from knowledge about the physics underlying the behaviour of the system.

Causal influences allow for representing causal dependency type knowledge. The Ca En formalism offers four types of influences whose internal form is presented later:

- dynamic, denoted by the symbol $-\mathrm{D} \rightarrow>$ between the influencing and the influenced variables;

integral, denoted by the symbol $-\mathrm{I}->$;

static, denoted by the symbol $-\mathrm{S}->$;

constant, denoted by the symbol $-\mathrm{C} \rightarrow>$.

Causal influences are characterized by several parameters, like a gain, a delay and a response time for dynamic influences, the gain and response time values can be given a real number or a real interval where known with imprecision. They also allow for a parameter condition, which specifies the logical conditions under which the influence is active. This is the key for representing hybrid systems.

The global constraint level is composed of functional numeric constraints associated with interval domains, e.g. constraints arising from physical laws. In other words, a global constraint is any algebraic equation, which may be non-linear, in which each unknown is assumed to take on interval values. This 
allows us to manage imprecise knowledge at this level as well. The global constraints are expressed by means of traditional arithmetic operators: $+,-, *, /$ and $* *$. These operators are interpreted in the interval algebra.

As variables and parameters take interval values, one can easily adapt the model's granularity to the requirements of the faults. Hence Ca En has a wide coverage of faults, from those radically changing the behaviour of the physical system to those causing smooth deviations.

\subsection{The Ca En prediction algorithm}

The prediction algorithm performs an estimation of the endogenous variable values across time. It can operate in an "open-loop mode", i.e. as a pure simulation, or in a "closedloop mode", i.e. by taking into account in real time the measured variable values and performing a reset. The temporal unit of the prediction module is the same as the data acquisition system. The input data are the causal model including initial conditions - and the evolution of the exogenous and other measured variables over time. The output of the system is the trajectory of each process variable [5]. The prediction module can be used on its own or coupled with the fault detection module, in which case it is used in a SCL mode as explained in section 3.3.

Predicting the variable values is one of the most critical steps in the interval model-based fault detection approach. The predictions need to be fine enough to be sensitive to faults, but not too fine so as to avoid generating false alarms.

In $\mathrm{Ca} \sim \mathrm{En}$, two steps are executed to predict the variable values: at the local constraint level and at the global constrain level.

(i) Local constraint level: Computation of The Updated Value of a Variable

From the superposition theorem that applies to the linear case, the computation of the updated value of a variable $y$ consists of processing the sum of the activated influences having exerted on the variable during the last time-interval.

Let's first consider the case in which $y$ is influenced by one variable only, say $x$, through an influence of a given type. Depending on its type, the Table 1 provides the internal form (discrete) of the influence used by Ca En as well as the continuous counterpart.

\begin{tabular}{|c|c|c|c|}
\hline Influence & $\begin{array}{c}\text { Representatio } \\
\text { n Formalism }\end{array}$ & $\begin{array}{c}\text { Transfer Function } \\
\text { (Diff. or Algebraic } \\
\text { equation) }\end{array}$ & $\begin{array}{c}\text { Discrete Transfer } \\
\text { Function }(\text { Ca } \sim \text { En } \\
\text { internal form) }\end{array}$ \\
\hline Dynamic & $x-\mathrm{D}->y$ & $\frac{Y(s)}{X_{i}(s)}=\frac{K e^{-s T_{d}}}{1+\tau s}$ & $\begin{array}{c}y(t+1)=a_{D} y(t)+ \\
b_{D} x_{i}(t-d)\end{array}$ \\
\hline Integral & $x-\mathrm{I}->y$ & $\frac{Y(s)}{X(s)}=\frac{K}{T s}$ & $\begin{array}{c}y(t+1)=a_{i} y(t)+ \\
b_{l} x_{i}(t-d)\end{array}$ \\
\hline Static & $x-\mathrm{S}->y$ & $\frac{Y(s)}{X_{i}(s)}=K$ & $y(t+1)=K x_{i}(t+1-d)$ \\
\hline Constant & $x-\mathrm{C}->y$ & $Y(s)=C$ & $y(t+1)=C$ \\
\hline
\end{tabular}

Table 1. Influences used by Ca En.
With the following agenda and equivalencies:

- $K$ is the gain;

- $\tau$ is the time constant of the transfer function which corresponds with good accuracy to $T_{r} / 3$, where $T_{r}$ is the response time;

- $T_{s}$ is the sampling period,

- $a_{D}=e^{-T_{s} / \tau} \approx e^{-3 T_{s} / T_{r}}$

- $a_{I}=1$

- $b_{D}=K\left(1-a_{D}\right)$.

- $b_{I}=K T_{s}$

Let's now generalize to a variable $y$ influenced (actively) by a set of variables $x_{i}, i=1, \ldots n$. The influences $i=1, \ldots, n$ may be of different type. Differential influences $i \in D$, integral influences $i \in I$, static $i \in S$ and constant influences $i \in C$ can combine. Every influence is first materialised by an intermediate variable which stands for its associated marginal influence (this step is not necessary for static and constant influences). The combination is then performed by adding up all the marginal influences by means of static influences, as illustrate in Figure 3.

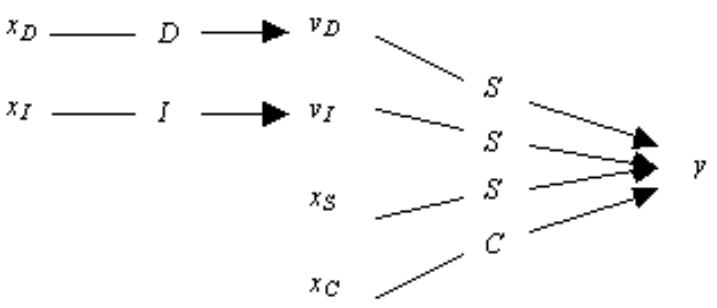

Figure 3. Ca En influence combination, $-X \rightarrow$ stands for a set of influences of type $X, X \in\{D, I, S, C\}$

The general recurrent equations used for updating the values of $v_{i}, i \in D, v_{j}, j \in I$, and $y$ at each sampling instant are given by:

$$
\left\{\begin{array}{c}
v_{i}(t+1)=a_{D i} v_{i}(t)+b_{D i} x_{i}\left(t-d_{i}\right) \quad i \in D \\
v_{j}(t+1)=a_{I j} v_{j}(t)+b_{I j} x_{j}\left(t-d_{j}\right) \quad j \in I \\
y(t+1)=\sum_{i \in D} v_{i}(t)+\sum_{j \in I} v_{j}(t)+\sum_{k \in S} K_{k} x_{k}\left(t-d_{k}\right)+\sum_{l \in C} C_{l}
\end{array}\right.
$$

where $a_{D i}, b_{D i}, a_{I j}$ and $b_{I j}$ are the dynamic and integral parameters specified above, $K_{k}$ are the gains of the static influences and $C_{l}$ the constants of the constant influences. Note that the delays and response times are automatically taken into account. The result is an interval.

\section{(ii) Global constraint level: Refinement of The Updated Variables' Values}

The numeric intervals obtained for the updated values (Equation (1)) are refined with the global constraints by performing a tolerance propagation algorithm [4] on the set of variables. The effect of the tolerance propagation algorithm is to filter (reduce) for consistency the values $y(t)$ using the global constraints.

The simulation results produced by the Ca En prediction module are envelopes (see Figure 4) for the variables of 
interest. The envelopes provide the upper and lower bound of the variable values at each sampled instant. As a consequence of the interval-based reasoning used in $\mathrm{Ca} \sim \mathrm{En}$, the results are complete but not correct.

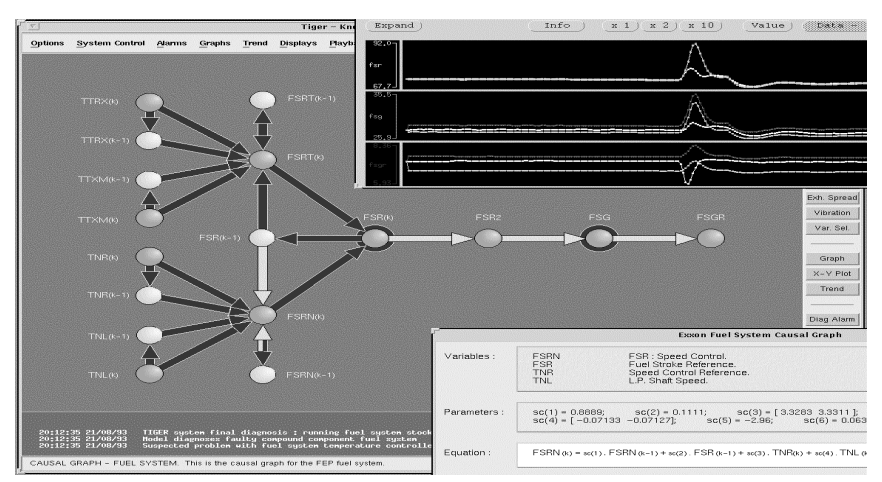

Figure 4: Ca En Causal Graph and Interval Predictions for the Fuel System Gas turbine.

\subsection{Ca En SCL fault detection strategy}

The Ca En fault detection procedure is based on models of normal behaviour. These are interval models, which capture imprecise knowledge as well as noise in the interval parameter values. The on line predictions obtained from these models is the basis of a discrepancy detection procedure based on adaptive thresholds, which allows us to track the physical system [8]. This is performed by comparing the predicted and observed values of variables across time so that static as well as dynamic discrepancies are detected. This is essential for controlled systems such as turbines. The controller indeed tends to compensate for the faults in such a way that the fault is only observable, and hence detectable, during the transient response of the turbine. The variables then stabilise at normal values. A classic limit checking diagnosis system is often inefficient in this kind of situation.

The ultimate goal of Ca En being to isolate the fault(s), a decoupling is performed at the level of every measured variable. This means that variable measured values are always used to determine the prediction for their downstream variables. For example, for a static influence, we have:

$$
y_{\text {pred }}(t+1)=K x_{\text {meas }}(t-d)
$$

At each instant $t$ and for every measured variable $y$, it is checked whether the measured value $y_{\text {meas }}(t)$ (a real number) belongs or not to the predicted value $y_{\text {pred }}(t)$ (an interval). If not, variable $y$ is said to be alarming at time $t$. This is equivalent to the calculation of an interval residual:

$$
r_{y}(t)=y_{\text {pred }}(t)-y_{\text {meas }}(t)
$$

where $r_{y}(t) \not \subset 0$ in the faulty case. Let's define as $A l$ the set of variables such that $r_{y}(t) \not \subset 0$. From the graphical point of view, this is interpreted as the observed trajectory going out of the predicted curve envelope at time $t$ as shown in Figure 5.

In practice, it may happen that noise in the measurements is not fully contemplated in the models or that the physical system is disturbed by other kinds of disturbances that have not been taken into account into the models. Consequently, a local incompatibility between prediction and observation at some instant $t$ does not necessarily mean that the system is faulty. In real applications, it is very important to have a robust fault detection system because a system which would untimely report faults would rapidly lose the confidence of the operator and engineering staff. Hence, we use a more robust indicator than just alarming variables. A fault is reported when a variable has remained alarming during a whole temporal interval $T$ of length significantly greater than the sampling period (cf. Figure 5). The variable is then said to be misbehaving. The length of $T$ may be regarded as a multiple of the sampling period, i.e. $T=v T_{s}$, and it should be adjusted according to the technology of the sensors (the choice of $v$ is left to the user).

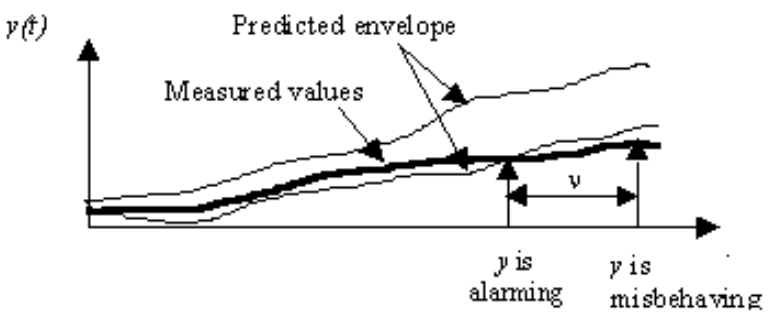

Figure 5: Fault detection is based on adaptive thresholds

More formally, the set MISB of misbehaving variables can be defined as follows:

$$
\begin{gathered}
y \in M I S B \text { at time } t \text { if } y \in A Z \text { since } t \text { - } v, \\
\text { i.e. } \forall i=0, \ldots, v, r_{y}(t-i) \not \subset 0
\end{gathered}
$$

Within the above presented framework, the Ca En fault detection strategy is a mixed strategy which combines an observer type strategy (closed-loop mode) with a simulation strategy (open-loop mode) to determine the residuals and further assess variable state. We call this strategy a semiclosed loop strategy SCL.

The mode control (open-loop or closed-loop) depends on whether the observed value of $y$ is in the predicted envelope (normal situation) or out of the predicted envelope (alarming situation) as follows:

If $y \notin A Z$ then closed-loop mode,

$$
y_{\text {pred }}(t+1)=a y_{\text {meas }}(t)+b x_{\text {meas }}(t-d)
$$

If $y \in A l$ then open-loop mode,

$$
y_{\text {pred }}(t+1)=a y_{\text {pred }}(t)+b x_{\text {meas }}(t-d)
$$

The two mentioned modes correspond to the schemas in Figure 6 (closed-loop mode) and Figure 7 (open-loop mode).

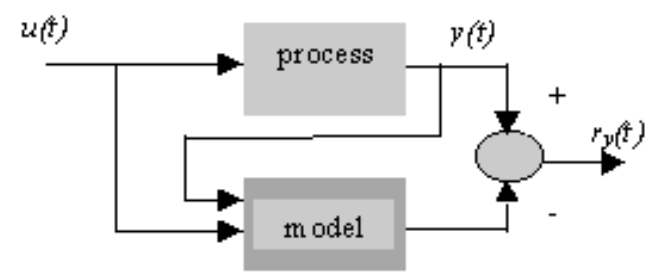

Figure 6. Close loop mode 


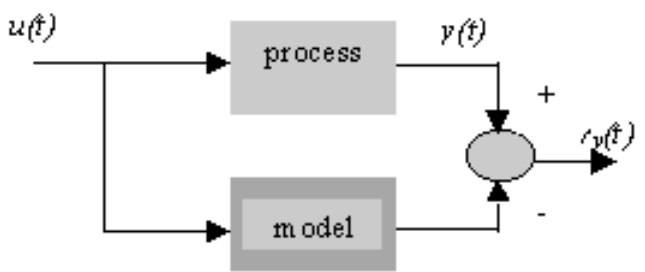

Figure 7. Open loop mode

The intuition behind this mixed strategy is related to two issues:

- $\quad$ The closed-loop mode runs on one step ahead predictions only, obtaining this way a good precision, which is critical when using interval models [1].

- As soon as the variable becomes alarming, running on a closed-loop mode would drive the prediction to follow the fault, turning the fault detection procedure insensitive to the fault.

\section{Qualitative causal models}

\subsection{Causal structure}

Given the measured exogenous variables and the intern measured variables of the GFS as well as the physical relations between them, a causal structure can be obtained [11]. A partial view of the causal structure is given in Figure 8. Others causal structures had been presented in [3].

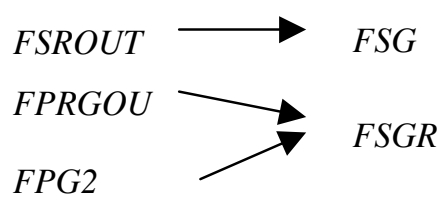

Figure 8. GFS (partial) causal structure

The isolation and detection operational model includes operational relations as:

$$
\begin{aligned}
& F S G R=f(F P G 2, \text { FPRGOUT }) \\
& F S G=\text { (FSROUT) }
\end{aligned}
$$

\subsection{Operational relations identification procedure}

The process of operational relation identification consists of several consecutive steps:

(i) Model structure selection: It is based on the a priori knowledge about the system and determines the success of the following steps.

(i) Model estimation (or parameter estimation): once the structure is selected, estimation algorithms process the experimental data from non faulty scenarios to determine the optimal interval values of the parameters. (ii) Model validation: this step verifies whether the obtained model is good enough for its intended use. If the obtained model does not satisfy the modelling requirements, the overall process is repeated in an iterative scheme. On the other side, if the model is assessed as good enough, the modelling process can be considered as completed. However, one may want to repeat the process again to obtain and compare along performance criteria different models based on different structures or different parameter estimation algorithms.

The interval parameters of a relation $\left.y=f x_{1}, \ldots, x_{n}, P\right)$, where $P$ is the parameter vector of dimension $m$, are tuned in two steps:

- Identification of nominal values. The least square algorithm provides nominal values for every parameter, $\hat{p}_{i} \in P$ and their statistical standard deviation, $\operatorname{std}\left(p_{i}\right)$.

- The interval values $\left[\hat{p}_{i}-\beta_{i_{1}} * \operatorname{std}\left(p_{i}\right), \hat{p}_{i}-\beta_{i_{2}} * \operatorname{std}\left(p_{i}\right)\right]$ are obtained using an iterative algorithm as follows:

$$
\begin{aligned}
& \text { For all } p_{i} \in P, \text { do } \\
& \begin{array}{l}
j=0, \beta_{i 1}=0, \beta_{i 2}=0, P^{0}=\hat{P}, y(t)=f\left(x_{1}, \cdots, x_{n}, \hat{P}\right) \\
\text { while } \quad \mathrm{y}_{\text {meas }}(t) \not \subset y_{j}(t) \quad \forall t \in \text { scenario } \\
j=j+1 \\
\forall i=1, \cdots, m \text { do } \\
\qquad p_{i}^{j}=\left[\hat{p}_{i}-\beta_{i 1}^{j} s t d\left(p_{i}\right), \hat{p}_{i}+\beta_{i 2}^{j} \operatorname{std}\left(p_{i}\right)\right] \\
\quad \beta_{i 1}^{j}=\beta_{i}^{j-1}{ }_{1}+\Delta, \beta_{i 2}^{j}=\beta_{i}^{j-1}{ }_{2}+\Delta \\
y_{j}(t)=f\left(x_{1}, \cdots, x_{n}, \hat{P}^{j}\right)
\end{array} \\
& \text { end }
\end{aligned}
$$

This algorithm expands the parameter interval values until the predicted envelope includes the experimental data.

The estimated operational relations in (4) are given by:

$$
F S G R(t)=F S G R(t-1)+K 1 \_G F S 3 *(F P G O U T(t-1)-F P G 2(t-1))+K 2 \_G F S 3
$$$$
F S G(t)=K \_G F S 1 * F S R O U T(t)
$$

where $K \_G F S 1, K 1 \_G F S 3$ and $K 2 \_G F S 3$ are interval values obtained from the above algorithm.

\section{Results}

The envelopes obtained for FSG and FSGR with (5) are shown in Figure 9 and 10. The figures show the expected high and low bounds of the envelope, as well as the actual value, for each variable. In addition, digital indications of whether the actual value is alarming or misbehaving according to the SCL strategy are available and can be displayed.

Figure 9 shows a normal actuator (GCV) behaviour, where the actual value of the valve position is always inside the envelope. Figure 9, corresponds to a faulty scenario where some abnormal spikes are present. 


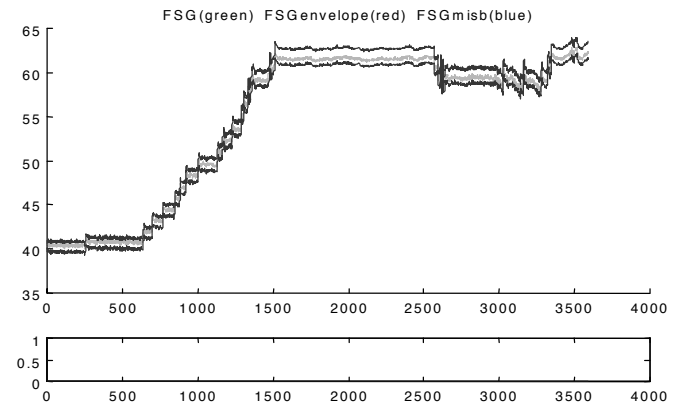

Figure 9. Non faulty scenario

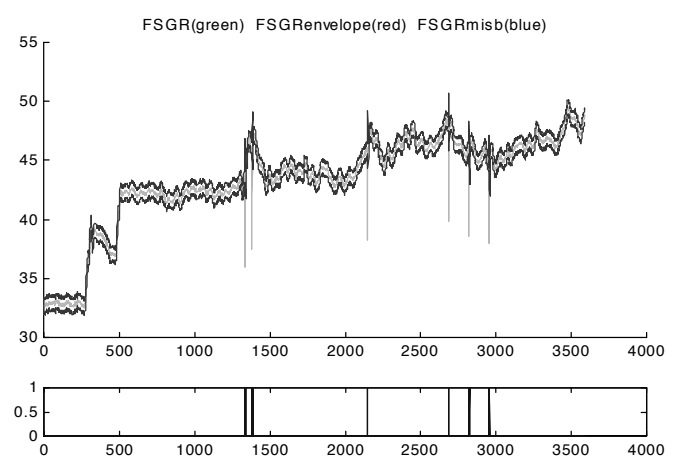

Figure 10. Faulty scenario

The above results have been obtained with a non robust SCL strategy $(v=1)$ to be able to capture the very fast abnormal spikes.

The SCL strategy (with $v=1$ ) has been compared to other detection strategies [2,6,7], and has shown good performances with respect to the false alarm/non detection compromise.

\section{Conclusions}

In this paper, the Ca En fault detection methodology based on the use of qualitative causal models with interval relationships has been described. The results of the application of this methodology to a real complex system, an industrial gas turbine, are also presented. In this application example, the estimated operational models give a fine output interval prediction based on a complex combination of inputs, which allows us to detect the spikes and unusual settings in a way that was not possible before, using static limit checking only.

Based on the efficient results obtained for such a complex system, the Ca En tool has been integrated in TIGER, and it is expected that this provides significant improvements in many other industrial turbines.

\section{Acknowledgements}

This paper is partially supported by the Spanish CICYT under contract TAP99-0748, by CIRIT of the Generalitat de Catalunya (ref. 1999SGR00134) and FP5 Research Training Network Proposal -ECC- TRN1-1999-00392.

\section{References}

[1] J. Armengol, L. Trave-Massuyes, J. Vehi, J.Ll. De La Rosa. "A survey on interval model simulators and their properties related to fault detection". Annual Reviews in Control, vol. 24, No. 1. ISSN: 1367-5788, (2000)

[2] J. Armengol, L.Trave-Massuyes, J. Vehi, M.A. Sainz. "Semiqualitative simulation using interval analysis. New perspectives for imprecise model-based fault detection" Proceedings of $14^{\text {th }}$ IFAC World Congress, pp. 521-526, (1999).

[3] T. Escobet, J. Quevedo, S. Tornil, L. Trave-Massuyes. "Integration of dynamic models in gas turbine supervision control”, 7th IEEE International Conference on Emerging Technologies and Factory Automation, pp. 995-1001, (1999).

[4] E. Hyvönen. "Constraint reasoning with incomplete knowledge; the tolerance propagation approach",Doctoral Dissertation, Technical Research Center of Finland, VTT Publications, Espoo, Finland (1992).

[5] R. Milne, L.Trave-Massuyes. "Model-based aspects of the TIGER gas turbine condition monitoring system", IFAC Symposium on Fault Detection, Supervision and Safety for Technical Processes, (1997).

[6] V. Puig, J. Saludes, J. Quevedo. "A new algorithm for adaptive threshold generation in robust fault detection based on a sliding window and global optimization". Proceedings of the European Control Conference, (1999).

[7] J. Quevedo, V. Puig, T. Escobet. "Model Fault Detection of Feedback Systems: How and Why to Use the Output of the PID Controller", Proceedings of the IFAC Workshop on Digital Control. Past, present and future of PID Control. pp. 363-368, (2000).

[8] S. Tornil, T. Escobet, V. Puig. "Fault Detection Using Interval Models", $4^{\text {th }}$ IFAC Symposium on Fault Detection, supervision and Safety for Technical Processes, pp. 1180-1186, (2000).

[9] L. Trave-Massuyes, R. Milne, "TIGER ${ }^{\mathrm{TM}}$ : Gas turbine condition monitoring using qualitative model based diagnosis", IEEE Expert Intelligent Systems \& Applications, volume 12, $\mathrm{n}^{\circ} 3$, pp. 22-31, (1997).

[10] L. Trave-Massuyes, J.A. Jimenez. "Fault detection and isolation in the Ca En system", LAAS-CNRS technical report (2001).

[11] L. Trave-Massuyes, R. Pons. "Causal ordering for multiple mode systems", 11th International Workshop on Qualitative Reasoning, 11p, (1997). 